Nat i onwi de survey of $r$ adi at $i$ on exposure during pedi at ri c comput ed tomography exam nat i ons and proposal of age- based di agnost i c ref er ence I evel s for Japan.

\begin{tabular}{|c|c|}
\hline 著者 & 竹井 泰孝 \\
\hline 著者別表示 & Takei Yasut aka \\
\hline $\begin{array}{l}\text { jour nal or } \\
\text { publ i cat i on title }\end{array}$ & 博士論文本文Ful I \\
\hline 学位授与番号 & 13301甲第4386号 \\
\hline 学位名 & 博士( 保健学) \\
\hline 学位授与年月日 & $2016-03-22$ \\
\hline URL & ht t p: //hdl . handl e. net /2297/45288 \\
\hline
\end{tabular}


Nationwide survey of radiation exposure during pediatric computed tomography examinations and proposal of age-based diagnostic reference levels for Japan

Yasutaka Takei, Osamu Miyazaki, Kosuke Matsubara, Yoshiya Shimada, Yoshihisa Muramatsu, Keiichi Akahane, Keisuke Fujii, et al.

Pediatric Radiology

ISSN 0301-0449

Pediatr Radiol

DOI 10.1007/s00247-015-3474-x
A 20197

ONLINE

FIRST

\section{Pediatric Radiology}

Official Journal of the

European Society of Paediatric Radiology

Society for

Pediatric Radiology

Asian and Oceanic

Society for

Pediatric Radiology

Latin American

Society of

Pediatric Radiology 
Your article is protected by copyright and all rights are held exclusively by SpringerVerlag Berlin Heidelberg. This e-offprint is for personal use only and shall not be selfarchived in electronic repositories. If you wish to self-archive your article, please use the accepted manuscript version for posting on your own website. You may further deposit the accepted manuscript version in any repository, provided it is only made publicly available 12 months after official publication or later and provided acknowledgement is given to the original source of publication and a link is inserted to the published article on Springer's website. The link must be accompanied by the following text: "The final publication is available at link.springer.com". 


\title{
Nationwide survey of radiation exposure during pediatric computed tomography examinations and proposal of age-based diagnostic reference levels for Japan
}

\author{
Yasutaka Takei $^{1}$ - Osamu Miyazaki ${ }^{2}$ Kosuke Matsubara ${ }^{3} \cdot$ Yoshiya Shimada $^{4}$. $^{\circ}$ \\ Yoshihisa Muramatsu $^{5} \cdot$ Keiichi Akahane $^{4} \cdot$ Keisuke Fujii $^{6}$ - Shoichi Suzuki ${ }^{7}$. \\ Kichiro Koshida ${ }^{3}$
}

Received: 6 April 2015 / Revised: 2 July 2015 / Accepted: 7 October 2015

(C) Springer-Verlag Berlin Heidelberg 2015

\begin{abstract}
Background Diagnostic reference levels (DRLs) have not been established in Japan.

Objective To propose DRLs for CT of the head, chest and abdomen for three pediatric age groups.

Materials and methods We sent a nationwide questionnaire by post to 339 facilities. Questions focused on pediatric CT technology, exposure parameters, CT protocols, and radiation doses for age groups $<1$ year, $1-5$ years, and 6-10 years.
\end{abstract}

Yasutaka Takei

ytakei-ham@umin.net

1 Department of Quantum Medical Technology,

Division of Health Sciences, Graduate School of Medical Science,

Kanazawa University,

5-11-80 Kodatsuno, Kanazawa, Ishikawa 920-0942, Japan

2 Department of Radiology,

National Center for Child Health and Development,

Tokyo, Japan

3 Department of Quantum Medical Technology,

Faculty of Health Sciences,

Kanazawa University,

Ishikawa, Japan

4 Medical Exposure Research Project,

National Institute of Radiological Sciences,

Chiba, Japan

5 Department of Radiology,

National Cancer Center Hospital East,

Chiba, Japan

6 Radiological Technology, Graduate School of Medicine, Nagoya University, Aichi, Japan

7 Faculty of Radiological Technology, School of Health Sciences, Fujita Health University,

Aichi, Japan
Results For the three age groups in the 196 facilities that responded, the 75th percentile values of volume CT dose index based on a $16-\mathrm{cm}$ phantom $\left(\mathrm{CTDI}_{\mathrm{vol}} 16[\mathrm{mGy}]\right)$ for head, chest and abdominal CT were for infants 39.1, 11.1 and 12.0, respectively; for 1-to 5-year-olds $46.9,14.3$ and 16.7, respectively; and for 6-to 10-year-olds 67.7, 15.0 and 17.0, respectively. The corresponding dose-length products (DLP 16 $[\mathrm{mGy} \cdot \mathrm{cm}])$ for head, chest and abdominal CT were for infants 526.1, 209.1 and 261.5, respectively; for 1-to 5-year-olds 665.5, 296.0 and 430.8, respectively; and for 6-to 10-yearolds $847.9,413.0$ and 532.2, respectively.

Conclusion The majority of CTDI $\mathrm{vol}_{\mathrm{vo}} 16$ and DLP 16 values for the head were higher than DRLs reported from other countries. For risk reduction, it is necessary to establish DRLs for pediatric CT in Japan.

Keywords Child · Computed tomography $\cdot$ Diagnostic reference levels $\cdot$ Patient exposure $\cdot$ Pediatric

\section{Introduction}

As a result of the East Japan earthquake on March 11, 2011, large amounts of radioactive substances were released into the environment by the Fukushima Daiichi nuclear power plant $[1,2]$. This accident has led to widespread public unease concerning radiation, and many hospitals have received numerous queries from patients with regard to medical radiation exposure.

Radiologists have a duty of providing accurate information regarding medical radiation exposure and explaining it to the public. Consequently, it is important to have current data that allow for global comparison and evaluation. A certain level of control is necessary over radiation exposure in patients; 
therefore the International Commission on Radiological Protection (ICRP) has recommended the use of diagnostic reference levels (DRLs) [3]. However in Japan DRLs have not been set for diagnostic radiology.

The purpose of this study was to conduct a nationwide questionnaire survey concerning radiation exposure during pediatric $\mathrm{CT}$ of the head, chest and abdomen, and to propose DRLs for three pediatric age groups: infants (ages $<1$ year), young children (ages 1-5 years) and children (ages 610 years) in Japan.

\section{Materials and methods}

\section{Ethics}

Institutional review board approval was not required for this retrospective nationwide questionnaire study, and this study did not require informed patient consent.

\section{Selection of study facilities}

We sent questionnaire forms - including a website address for posting responses - by post to 339 facilities of members (as of March 2012) of the Japanese Society of Radiological Technology. These were mainly university and national hospitals that form the core of community medicine in Japan. We received responses to the questionnaire by post or through the website. We obtained approval from each facility to collect responses to the questionnaire by including this sentence: "Your response will not be used for anything other than calculating and analyzing the radiation dose and will be managed appropriately" in the questionnaire form.

\section{Survey items}

In the questionnaire, parameters regarding the scanning conditions included the assessment criteria for the pediatric $\mathrm{CT}$ protocol. These included tube voltage, tube current time product and rotation time, volume CT dose index $\left(\mathrm{CTDI}_{\mathrm{vol}}\right)$ and dose-length product (DLP) displayed on the CT systems during head, chest and abdominal CT. The children were classified into three age groups: infants (ages $<1$ year), young children (ages $1-5$ years) and children (ages 6-10 years). We asked participating institutions to confirm that the displayed $\mathrm{CTDI}_{\mathrm{vol}}$ was based on a $16-\mathrm{cm}$ phantom. For devices that displayed the $\mathrm{CTDI}_{\mathrm{vol}}$ based on a 32-cm phantom, the participants were asked to enter the displayed $\mathrm{CTDI}_{\mathrm{vol}} 32$, which was explicitly stated in the form. We converted CTDI ${ }_{\mathrm{vol}} 32$ to $\mathrm{CTDI}_{\mathrm{vol}} 16$ by multiplying by a factor of $2[4,5]$.

\section{Data analysis}

The CT scanning conditions, CTDI ${ }_{\mathrm{vol}}$ and DLP, obtained from the questionnaire were summarized and compared among facilities. Statistical significance was determined using Student's $t$-test, and the significance level was set at $P<0.05$. Calculations were performed using Microsoft Excel version 2010 (Microsoft, Redmond, WA).

\section{Results}

Of the 339 facilities to which the questionnaires were sent, people at $196(58 \%)$ responded. In total, 1,002 displayed CTDI $_{\text {vol }}$ values from 164 facilities and 955 displayed DLP values from 157 facilities were available for data analysis. This was because 32 cases involving CTDI $_{\mathrm{vol}}$ and 39 cases involving DLP were excluded because participants entered values incorrectly, the entry form could not be corrected, or there were blank entries on the form.

\section{Tube voltage}

For all age groups and scanned areas, the most frequently used tube voltage was $120 \mathrm{kV}$, which was used in $90 \%$ (522/578) of head CT scans, $79 \%(265 / 334)$ of chest CT scans and $82 \%$ (277/339) of abdominal CT scans. A low tube voltage of 80 $100 \mathrm{kV}$ was not used frequently; it was only used in 7\% (42/ 578 ) of head CT scans, $18 \%(61 / 334)$ of chest CT scans, and $16 \%(55 / 339)$ of abdominal CT scans.

\section{Tube current time product (mAs)}

The median value of the tube current time ranged 120-225 mAs for the head, 46-63 mAs for the chest and 50-75 mAs for the abdomen (Fig. 1). Current-time products increased with age.

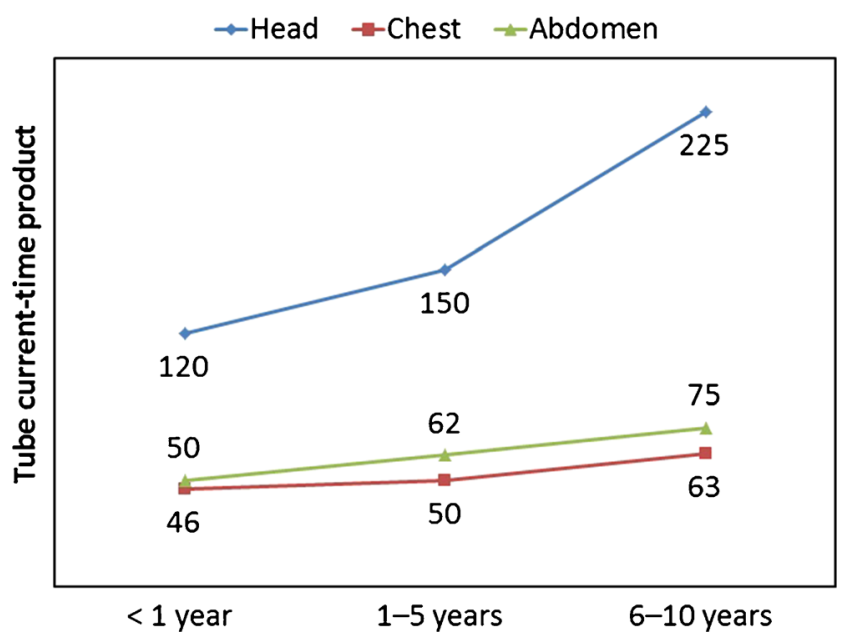

Fig. 1 Median tube current-time product for head, chest and abdominal CT scans by age group based on data from the Japanese national survey 


\section{Rotation time}

For head examination, the most frequent rotation time used for children of all age groups was $1.0 \mathrm{~s}$ (41-48\% of protocols) followed by $0.5 \mathrm{~s}$ (17-29\% of protocols). For the chest, $0.5 \mathrm{~s}$ was used in $50-65 \%$ of protocols, $0.4 \mathrm{~s}$ in $20-30 \%$, and $0.3 \mathrm{~s}$ in $1-7 \%$. For the abdomen, $0.5 \mathrm{~s}$ was used in $65-73 \%$ of protocols, $0.4 \mathrm{~s}$ in $17-23 \%$, and $0.3 \mathrm{~s}$ in $1-4 \%$.

\section{Pitch values}

The pitch values were reported between 0.3 and 1.6 for different examinations and age groups. For head examination, a pitch of $\leq 1$ was used in $94-97 \%$ of protocols, depending on the age groups. The most frequent pitch for all age groups was 0.7 , used in $38-44 \%$ of protocols. For chest examination, a pitch between 0.9 and 1.6 was used in $89-91 \%$ of protocols, depending on the age group. The most frequent pitch for all age groups was 0.9 , used in $31-35 \%$ of protocols. Similarly, for the abdominal examination, a pitch between 0.9 and 1.6 was used in $85-92 \%$ of protocols. The most frequent pitch for all age groups was 0.9 , used in $22-24 \%$ of protocols.

\section{Displayed CTDI ${ }_{\text {vol }}$ on CT systems}

Nine hundred seventy displayed $\mathrm{CTDI}_{\mathrm{vol}}$ values were obtained at 164 facilities. In the infant group, 16\% (152/970), 8\% (79/970) and 8\% (74/970) were for head, chest and abdominal CT scans, respectively. In the young-children group, $16 \%$ (154/970), 8\% (81/970) and 9\% (84/970) were for head, chest and abdominal CT scans, respectively. In the children group, 17\% (164/970), 9\% (89/970) and 10\% (93/970) were for head, chest and abdominal CT scans, respectively.

Table 1 shows minimum, maximum, median and 75 th percentile values of $\mathrm{CTDI}_{\mathrm{vol}}$ from routine protocols. The 75 th percentile $\mathrm{CTDI}_{\mathrm{vol}}[\mathrm{mGy}]$ ranged $39.1-67.7$ for the head,

Table 1 The minimum, maximum, median and 75th percentile values of the computed tomography dose index volume $\left(\mathrm{CTDI}_{\mathrm{vol}}\right)$ from routine protocols in three pediatric age groups

\begin{tabular}{llllll}
\hline $\begin{array}{l}\mathrm{C}_{\text {TDI }} \text { vol } \\
\text { (mGy) }\end{array}$ & Age group & Minimum & Maximum & Median & $\begin{array}{l}\text { 75th } \\
\text { percentile }\end{array}$ \\
\hline Head & $<$ 1 year & 9.4 & 120.0 & 30.7 & 39.1 \\
& 1-5 years & 9.4 & 109.3 & 36.1 & 46.9 \\
& 6-10 years & 6.1 & 155.3 & 47.8 & 67.7 \\
Chest & $<1$ year & 0.6 & 48.0 & 5.4 & 11.1 \\
& 1-5 years & 1.0 & 48.0 & 7.7 & 14.3 \\
& 6-10 years & 2.2 & 33.9 & 8.3 & 15.0 \\
Abdomen & $<1$ year & 0.9 & 46.9 & 6.4 & 12.0 \\
& 1-5 years & 1.5 & 46.9 & 9.7 & 16.7 \\
& 6-10 years & 1.6 & 33.9 & 10.0 & 17.0 \\
\hline
\end{tabular}

11.1-15 for the chest, and $12-17$ for the abdomen. The $\mathrm{CTDI}_{\mathrm{vol}}$ values increased with age.

\section{Dose-length product displayed on CT systems}

Nine hundred sixteen displayed DLP values were obtained at 157 facilities. In the infant group, 16\% (143/916), 8\% (73/ 916) and 7\% (68/916) were for head, chest and abdominal CT scans, respectively. In the young-children group, $16 \%$ (145/916), 8\% (76/916) and 9\% (79/916) were for head, chest and abdominal CT scans, respectively. In the children group, $17 \%$ (157/916), 9\% (85/916) and 10\% (90/916) were for head, chest and abdominal CT scans, respectively.

Table 2 shows the minimum, maximum, median and 75th percentile values of DLP obtained from routine protocols. The 75th percentile DLP values [ $\mathrm{mGy} \cdot \mathrm{cm}$ ] ranged 526.1-847.9 for the head, 209.1-413.0 for the chest and 261.5-532.2 for the abdomen. The DLP values increased with age.

\section{Comparison of 75th percentile of $\mathrm{CTDI}_{\mathrm{vol}}$ and dose-length product values with other surveys}

Tables 3 and 4 show the comparison between $\mathrm{CTDI}_{\mathrm{vol}}$ and DLP values obtained in the present study with those from other published surveys from the United Kingdom, Germany, Switzerland, Thailand and the International Atomic Energy Agency (IAEA) [6-10]. All age-based 75th percentiles of the $\mathrm{CTDI}_{\mathrm{vol}}$ values for the head were 1-2 times higher and DLP values for the head were 0.9-1.9 times higher in the Japanese survey than in the other published surveys. The 75th percentile of the $\mathrm{CTDI}_{\mathrm{vol}}$ values for the chest were 1.4 3.2 times higher and the DLP values for the chest were 1.4 3.8 times higher in the Japanese survey than those reported from the German, Swiss, Thai and French surveys [7-9, 11] and nearly equal to the results reported for the British and IAEA surveys [6-10]. The 75 th percentile of the CTDI ${ }_{\mathrm{vol}}$

Table 2 The minimum, maximum, median and 75th percentile values of the dose-length product (DLP) obtained from routine protocols

\begin{tabular}{llllll}
\hline $\begin{array}{l}\text { DLP } \\
(\mathrm{mGy} \cdot \mathrm{cm})\end{array}$ & Age group & Minimum & Maximum & Median & $\begin{array}{l}\text { 75th } \\
\text { percentile }\end{array}$ \\
\hline Head & $<1$ year & 13.0 & $2,066.0$ & 398.4 & 526.1 \\
& 1-5 years & 16.7 & $2,066.0$ & 463.5 & 665.5 \\
& 6-10 years & 16.7 & $2,841.5$ & 593.6 & 847.9 \\
Chest & $<1$ year & 11.1 & 945.4 & 90.0 & 209.1 \\
& 1-5 years & 17.5 & 945.4 & 159.9 & 296.0 \\
& 6-10 years & 24.0 & 960.3 & 228.6 & 413.0 \\
Abdomen & $<1$ year & 12.4 & $1,980.0$ & 153.5 & 261.5 \\
& $1-5$ years & 54.8 & $1,980.0$ & 251.0 & 430.8 \\
& 6-10 years & 47.7 & $1,980.0$ & 275.5 & 532.2 \\
\hline
\end{tabular}


Table 3 The 75th percentiles of the computed tomography dose index volume $\left(\mathrm{CTDI}_{\mathrm{vol}}\right)$ for pediatric CT scans in this study, by age and body part, compared with other surveys

\begin{tabular}{|c|c|c|c|c|c|c|c|c|c|}
\hline \multirow[t]{2}{*}{ 75th percentile of $\mathrm{CTDI}_{\mathrm{vol}}(\mathrm{mGy})$} & \multicolumn{3}{|l|}{ Head } & \multicolumn{3}{|l|}{ Chest } & \multicolumn{3}{|c|}{ Abdomen } \\
\hline & $<1$ year & $1-5$ years & $6-10$ years & $<1$ year & $1-5$ years & $6-10$ years & $<1$ year & $1-5$ years & $6-10$ years \\
\hline Japan (this study) & 39.1 & 46.9 & 67.7 & 11.1 & 14.3 & 15 & 12 & 16.7 & 17 \\
\hline IAEA [10] & 29 & 37.7 & 46.1 & ${ }^{\mathrm{a}} 14$ & ${ }^{\mathrm{a}} 16.4$ & ${ }^{\mathrm{a}} 20$ & ${ }^{\mathrm{a}} 21.4$ & ${ }^{\mathrm{a}} 26$ & ${ }^{\mathrm{a}} 24$ \\
\hline Thailand [9] & 26 & 29 & 39 & 4.5 & 5.7 & 10 & 7.7 & 8.9 & 13.8 \\
\hline France [11] & 30 & 40 & 50 & ${ }^{\mathrm{a}} 6$ & $\mathrm{a}_{7}$ & ${ }^{\mathrm{a}} 11$ & ${ }^{\mathrm{a}} 8$ & ${ }^{\mathrm{a}} 9$ & ${ }^{\mathrm{a}} 14$ \\
\hline Switzerland [8] & 20 & 30 & 40 & 5 & 8 & 10 & 7 & 9 & 13 \\
\hline Germany [7] & 33 & 40 & 50 & 3.5 & 5.5 & 8.5 & 5 & 8 & 13 \\
\hline United Kingdom [6] & 30 & 45 & 50 & 12 & 13 & 20 & 20 & 20 & 30 \\
\hline
\end{tabular}

${ }^{\mathrm{a}}$ Converted $\mathrm{CTDI}_{\mathrm{vol} 16}$ by doubling $\mathrm{CTDI}_{\mathrm{vol} 32}$

values for the abdomen were 1.2-2.4 times higher and the DLP values for the abdomen were 1.1-2 times higher in the Japanese survey than in the German, Swiss, Thai and French surveys $[7-9,11]$ and lower than in the British and IAEA surveys $[6-10]$.

\section{Relationship between tube voltage and the displayed CTDI $_{\text {vol }}$}

The relationship between the tube voltage and 75th percentile of the displayed CTDI ${ }_{\mathrm{vol}}$ for the head, chest and abdominal CT scans in each age group is shown in Figs. 2, 3, and 4. The Student's $t$-test for statistical significance was performed between the tube voltage and displayed $\mathrm{CTDI}_{\mathrm{vol}}$ for the head, chest and abdominal CT scans in each age group. For all scanning areas and all age groups, a significant difference of $P<0.01$ was observed between the CTDI ${ }_{\mathrm{vol}}$ for a tube voltage of $80 \mathrm{kV}$ and that for a tube voltage of $120 \mathrm{kV}$.

Table 5 compares the $\mathrm{CTDI}_{\mathrm{vol}}$ and DLP values proposed as DRLs for pediatric CT in Japan to the CTDI $\mathrm{I}_{\mathrm{vol}}$ and DLP for head, chest, and abdominal CT in each age group obtained from our survey.

\section{Discussion}

The aim of this study was to conduct a nationwide survey of radiation exposure during pediatric $\mathrm{CT}$ examinations and to establish DRLs in Japan. The survey conducted by our research group has revealed, for the first time, the details of pediatric $\mathrm{CT}$ radiation exposure in Japan such as the scanning conditions, $\mathrm{CTDI}_{\mathrm{vol}}$ and dose-length product. This survey was carried out in relatively large medical facilities, such as university and national hospitals, which form the core of community medicine in Japan. Consequently, it is likely that the results are an accurate representation of pediatric $\mathrm{CT}$ activities in Japan.

Many facilities are using a tube voltage of $120 \mathrm{kV}$ as a scanning condition for pediatric CT in Japan - $90 \%$ of the facilities surveyed used this voltage. In contrast, only a few facilities are using a low tube voltage of $80-100 \mathrm{kV}$, which is useful for reducing pediatric $\mathrm{CT}$ radiation exposure [12]. When we investigated the relationship between the tube voltage and the $\mathrm{CTDI}_{\mathrm{vol}}$, we found that the $\mathrm{CTDI}_{\mathrm{vol}}$ for facilities using a tube voltage of $80 \mathrm{kV}$ was significantly lower than that for facilities using $120 \mathrm{kV}$. Using a low tube voltage, the $\mathrm{CTDI}_{\mathrm{vol}}$ can be reduced in pediatric CT. When considering

Table 4 Comparison of 75th percentile of the dose-length product (DLP) for pediatric CT scans in this study, by age and body part, compared with other surveys

\begin{tabular}{|c|c|c|c|c|c|c|c|c|c|}
\hline \multirow[t]{2}{*}{ 75th percentile of DLP $(\mathrm{mGy} \cdot \mathrm{cm})$} & \multicolumn{3}{|l|}{ Head } & \multicolumn{3}{|l|}{ Chest } & \multicolumn{3}{|c|}{ Abdomen } \\
\hline & $<1$ year & $1-5$ years & $6-10$ years & $<1$ year & $1-5$ years & $6-10$ years & $<1$ year & $1-5$ years & $6-10$ years \\
\hline Japan (this study) & 526 & 666 & 848 & 209 & 296 & 413 & 262 & 431 & 532 \\
\hline Thailand [9] & 402 & 570 & 613 & 80 & 140 & 305 & 222 & 276 & 561 \\
\hline France [11] & 420 & 600 & 900 & ${ }^{\mathrm{a}} 60$ & ${ }^{\mathrm{a}} 126$ & ${ }^{\mathrm{a}} 274$ & ${ }^{\mathrm{a}} 160$ & ${ }^{a} 242$ & a 490 \\
\hline Switzerland [8] & 270 & 420 & 560 & 110 & 200 & 220 & 130 & 300 & 380 \\
\hline Germany [7] & 390 & 520 & 710 & 55 & 110 & 210 & 145 & 255 & 475 \\
\hline United Kingdom [6] & 270 & 470 & 620 & 200 & 230 & 370 & 330 & 360 & 800 \\
\hline
\end{tabular}

${ }^{\text {a }}$ Converted to $\mathrm{DLP}_{16}$ by doubling $\mathrm{DLP}_{32}$ 


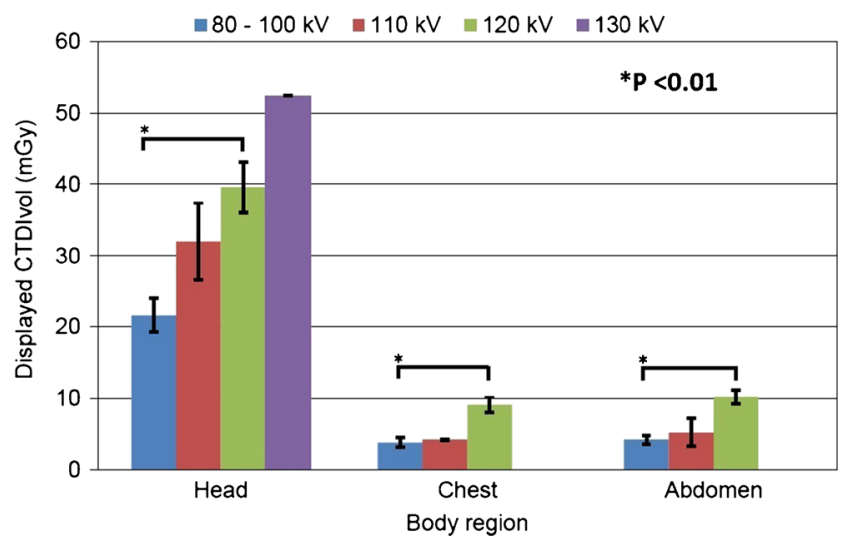

Fig. 2 Relationship between the CT tube voltage and the 75th percentile displayed as the $\mathrm{CTDI}_{\mathrm{vol}}$ on CT systems in head, chest and abdominal CT scans in infants $(P<0.01)$

the optimization of exposure in pediatric CT, the use of low tube voltage is an important feature.

Previous studies on radiation exposure in pediatric patients, who are particularly vulnerable to the effects of radiation, have been conducted $[13,14]$; these studies focused on calculation of the population dose and the evaluation of exposure in terms of mAs. Fukushima et al. [15] conducted a survey on the radiation exposure involved in CT examination within Gunma Prefecture and reported that the DLP for pediatric head CT was higher than that used in other countries. Thus, pediatric CT radiation exposure in Japan was expected to be higher than that in other countries.

Our survey also found that the DLP values for pediatric CT in Japan were higher than in other surveys [8-11]. The 75th percentile values of the $\mathrm{CTDI}_{\mathrm{vol}}$ for pediatric $\mathrm{CT}$ in our survey were higher than those in the survey conducted by the IAEA [10]. Furthermore, the 75th percentile values of DLP were markedly higher than those reported in other countries [8-11]. In other words, our results imply that $\mathrm{CTDI}_{\mathrm{vol}}$ of pediatric CT is higher in Japan than in other countries and that scanning is being conducted over a wider area of the body.

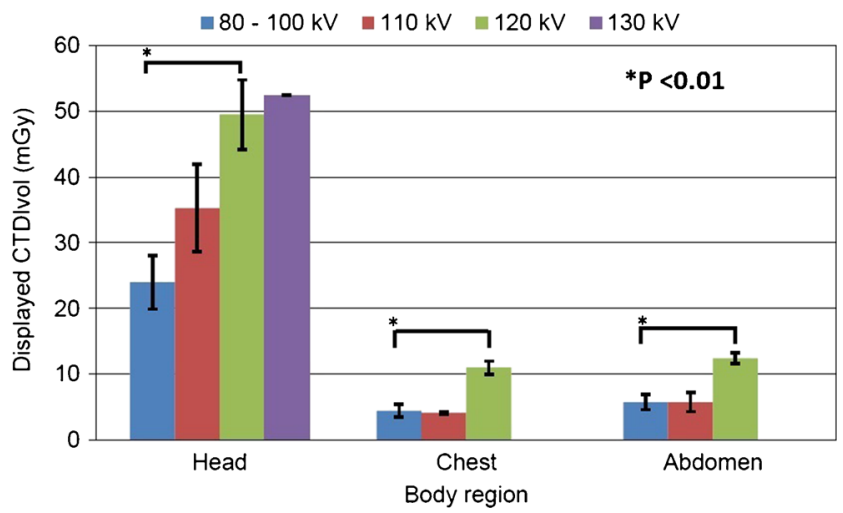

Fig. 3 Relationship between the CT tube voltage and the 75th percentile displayed as the $\mathrm{CTDI}_{\mathrm{vol}}$ on CT systems in head, chest and abdominal CT scans in 1-to 5-year-olds $(P<0.01)$



Fig. 4 Relationship between the scanning tube voltage and the 75th percentile displayed as the $\mathrm{CTDI}_{\mathrm{vol}}$ on CT systems in head, chest and abdominal CT scans in 6-to 10-year-olds

In Japan, pediatric CT protocols are assessed according to image quality and dose. However, many facilities use other quality assessments such as consultation with a physician and assessments based on experience and manufacturer recommendations [16]. No clear standard on image quality for pediatric $\mathrm{CT}$ has been established; therefore the scanning conditions adopted have been at the discretion of the physician or radiologic technologist at the clinical site. Consequently, the standard scanning conditions for pediatric CT scans have not necessarily been set appropriately. Thus there is room for improvement in optimizing the balance between image quality and radiation exposure in pediatric CT examination in Japan. Radiologic staff training has been found to be effective in reducing radiation exposure [17]. Therefore it is necessary to carry out appropriate education for radiologic staff in Japan regarding radiation exposure in pediatric $\mathrm{CT}$.

Regarding our survey results, we excluded 32 cases involving $\mathrm{CTDI}_{\mathrm{vol}}$ and 39 cases involving DLP because participants entered values incorrectly, the entry form could not be corrected, or there were blank entries on the form. We received responses from 196 facilities; therefore after exclusions we had CTDI $_{\mathrm{vol}}$ values from 164 facilities and the DLPs from

Table 5 The CTDIvol and DLP values proposed as age-based DRLs for pediatric CT scans in Japan

\begin{tabular}{lllll}
\hline Body region & Proposed DRLs & $<1$ year & $1-5$ years & $6-10$ years \\
\hline Head & CTDI $_{\text {vol }}(\mathrm{mGy})$ & 38 & 47 & 60 \\
& DLP $(\mathrm{mGy} \cdot \mathrm{cm})^{*}$ Chest & 500 & 660 & 850 \\
& CTDI $_{\mathrm{vol}}(\mathrm{mGy})$ & $11(5.5)$ & $14(7)$ & $15(7.5)$ \\
Abdomen & DLP $(\mathrm{mGy} \cdot \mathrm{cm})$ & $210(105)$ & $300(150)$ & $410(205)$ \\
& CTDI $_{\mathrm{vol}}(\mathrm{mGy})$ & $11(5.5)$ & $16(8)$ & $17(8.5)$ \\
& DLP $(\mathrm{mGy} \cdot \mathrm{cm})$ & $220(110)$ & $400(200)$ & $530(265)$ \\
\hline
\end{tabular}

The numbers in brackets are phantom measurements $(32-\mathrm{cm}$ diameter PMMA phantom) 
157 facilities for data analysis. However, $57 \%$ of the facilities did not provide data for at least one of the scanning conditions, $\mathrm{CTDI}_{\mathrm{vol}}$ or DLP, and for at least one of the age groups, which was the principal limitation of our questionnaire survey. The latest multi-detector CT systems often have a function to output $\mathrm{CTDI}_{\mathrm{vol}}$ and DLP values in the form of a radiation dose structure report formed by digital imaging and communications in medicine [18]. In the United States, this dose report is used for dose optimization through a dose index registry via the dose index reporting application [19]. The introduction of the registry using these dose reports in the future would facilitate the collection of more precise CTDI $_{\mathrm{vol}}$ and DLP data from many facilities in Japan.

Based on the distribution of the $\mathrm{CTDI}_{\mathrm{vol}}$ for pediatric CT gathered from this survey, we have proposed DRLs for pediatric CT examinations in Japan. Although our proposed DRLs are higher than those used in other countries, it is important to encourage facilities that are conducting examinations using $\mathrm{CTDI}_{\mathrm{vol}}$ that are higher than the values that we have proposed to urgently reassess their scanning conditions. Regarding practical application, the results obtained in the current survey can contribute to the prompt establishment of DRLs for pediatric $\mathrm{CT}$ examinations to promote the optimization of pediatric CT scan protocols in Japan.

\section{Conclusion}

Our survey of pediatric CT in Japan showed that all age-based Japanese 75th percentiles of the CTDI $\mathrm{vol}_{\mathrm{vol}}$ and DLP values were higher than in surveys of other countries. To promote the optimization of pediatric $\mathrm{CT}$ scan protocols, it is therefore necessary to establish DRLs for pediatric CT examinations in Japan.

Acknowledgments We thank the members of Japanese Society of Radiological Technology for their cooperation in the questionnaire survey, and Mr. Yuta Matsunaga and Ms. Ai Kawaguchi for their assistance in sending and collecting the questionnaire. This research was financially supported by the Japanese Society of Radiological Technology (Scientific Research Group 2012 grant) and a Japanese National Center for Child Health and Development (grant 26-20).

\section{Compliance with Ethical Standards}

Conflicts of interest None

\section{References}

1. Hashimoto S, Ugawa S, Nanko K et al (2012) The total amounts of radioactively contaminated materials in forests in Fukushima, Japan. Sci Rep 2:416
2. Ueda S, Hasegawa H, Kakiuchi H et al (2013) Fluvial discharges of radiocaesium from watersheds contaminated by the Fukushima Dai-ichi Nuclear Power Plant accident, Japan. J Environ Radioact 118:96-104

3. International Commission on Radiological Protection (1996) Radiological protection and safety in medicine. ICRP Publication 73. Annals of ICRP(26). Pergamon Press, Oxford

4. Thomas KE, Wang B (2008) Age-specific effective doses for pediatric MSCT examinations at a large children's hospital using DLP conversion coefficients: a simple estimation method. Pediatr Radiol 38:645-656

5. McNitt-Gray MF (2002) AAPM/RSNA physics tutorial for residents: topics in CT. Radiation dose in CT. Radiographics 22: $1541-1553$

6. Shrimpton PC, Hillier MC, Lewis MA et al (2006) National survey of doses from CT in the UK: 2003. Br J Radiol 79:968-980

7. Galanski M, Nagel HD, Stamm G (2006) Paediatric CT exposure practice in the Federal Republic of Germany: results of a nation-wide survey in 2005/2006. Medizinishe Hochshule, Hannover

8. Verdun FR, Gutierrez D, Vader JP et al (2008) CT radiation dose in children: a survey to establish age-based diagnostic reference levels in Switzerland. Eur Radiol 18:1980-1986

9. Kritsaneepaiboon S, Trinavarat P, Visrutaratna P (2012) Survey of pediatric MDCT radiation dose from university hospitals in Thailand: a preliminary for national dose survey. Acta Radiol 53: $820-826$

10. Vassileva J, Rehani MM, Applegate K et al (2013) IAEA survey of paediatric computed tomography practice in 40 countries in Asia, Europe, Latin America and Africa: procedures and protocols. Eur Radiol 23:623-631

11. Brisse HJ, Aubert B (2009) CT exposure from pediatric MDCT: results from the 2007-2008 SFIPP/ISRN survey. J Radiol 90:207215

12. Nievelstein RA, van Dam IM, van der Molen AJ (2010) Multidetector CT in children: current concepts and dose reduction strategies. Pediatr Radiol 40:1324-1344

13. Nishizawa K, Matsumoto M, Iwai K et al (2004) Survey of CT practice in Japan and collective effective dose estimation. Nippon Igaku Hoshasen Gakkai Zasshi 64:151-158

14. Miyazaki O, Kitamura M, Masaki H et al (2005) Current practice of pediatric MDCT in Japan: survey results of demographics and agebased dose reduction. Nippon Igaku Hoshasen Gakkai Zasshi 65: 216-223

15. Fukushima Y, Tsushima Y, Takei H et al (2012) Diagnostic reference level of computed tomography (CT) in Japan. Radiat Prot Dosimetry 151:51-57

16. Takei Y, Suzuki S, Miyazaki O et al (2014) Summary of the survey on radiation exposure of pediatric CT examination in Japan focus on environments of CT examination. Nippon Hoshasen Gijutsu Gakkai Zasshi 70:562-568

17. Paolicchi F, Faggioni L, Bastiani L et al (2014) Optimization the balance between radiation dose and image quality in pediatric head $\mathrm{CT}$ : findings before and after intensive radiologic staff training. AJR Am J Roentgenol 202:1309-1315

18. Shih G, Lu ZF, Zabih R et al (2011) Automated framework for digital radiation dose index reporting from $\mathrm{CT}$ dose reports. AJR Am J Roentgenol 197:1170-1174

19. Morin RL, Coombs LP, Chatfield MB (2011) ACR dose index registry. J Am Coll Radiol 8:288-291 